\title{
NPM1 Gene Mutation
}

National Cancer Institute

\section{Source}

National Cancer Institute. NPM1 Gene Mutation. NCI Thesaurus. Code C82429.

Mutation of the nucleophosmin gene. It is seen in acute myeloid leukemias usually associated with a normal karyotype. 